\title{
Recent applications of the stochastic variational method
}

K. $\operatorname{Varga}^{1}{ }^{*}$, J. Usukura ${ }^{2}$, Y. Suzuki ${ }^{3}$

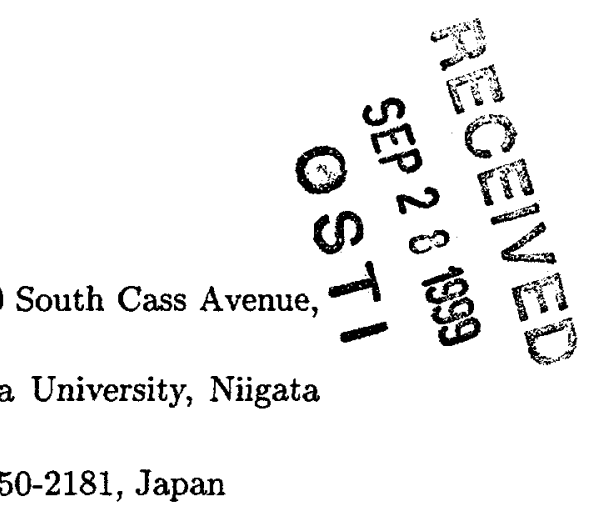

Abstract. This paper overviews the most recent developments and applications of the stochastic variational method for different physical systems.

\section{Introduction}

The variational method is a powerful and conceptually simple tool to solve the Schrödinger-equation. The wave function of the physical system is approximated by a linear combination of some appropriate "basis/trial" functions. The trial function may depend on some (continuous) parameters as well as on some quantum numbers (spins, isospins, orbital momentum, etc.) As the number of particles increases the selection of the most adequate trial function becomes more and more complicated. In principle one should optimize the wave function, but this way is not tractable (partly because the large number of parameters involved, partly because of the discreate variables (quantum numbers)).

The stochastic variational method (SVM) [1-13] attempts to find the most suitable basis functions by a simple trial and error gambling strategy. Several possible parameter and quantum number sets are probed and those which considerably improve the variational energy are selected to be basis states. The main idea is best explained by a simple example. We consider a simple yet nontrivial case: the Coulombic three-body system of two electrons and a positron.

* On leave from: Institute for Nuclear Research of the Hungarian Academy of Sciences (ATOMKI), 4001 Debrecen, PO Box 51, Hungary 


\section{DISCLAIMER}

This report was prepared as an account of work sponsored by an agency of the United States Government. Neither the United States Government nor any agency thereof, nor any of their employees, make any warranty, express or implied, or assumes any legal liability or responsibility for the accuracy, completeness, or usefulness of any information, apparatus, product, or process disclosed, or represents that its use would not infringe privately owned rights. Reference herein to any specific commercial product, process, or service by trade name, trademark, manufacturer, or otherwise does not necessarily constitute or imply its endorsement, recommendation, or favoring by the United States Government or any agency thereof. The views and opinions of authors expressed herein do not necessarily state or reflect those of the United States Government or any agency thereof. 


\section{DISCLAIMER}

Portions of this document may be illegible in electronic image products. Images are produced from the best available original document. 
The basis function, a correlated Gaussian $[14,15]$, is assumed in the form:

$$
f(A, \mathbf{x})=\exp \left\{-\frac{1}{2} \mathbf{x} A \mathbf{x}\right\}=\exp \left\{-\frac{1}{2} \sum_{i=1}^{2} \sum_{j=1}^{2} A_{i j} \mathbf{x}_{i} \cdot \mathbf{x}_{j}\right\}
$$

where $x_{1}$ and $x_{2}$ are the relative coordinates between the two electrons, and between the center-of-mass of the two electrons and the positron, respectively. The matrix elements $A_{i j}$ are the parameters of the trial function. This basis function belongs to $L=0$ orbital motion, and electrons have antiparallel spins. As only Coulomb interaction is considered between the particles there is no additional quantum number needed to describe the systems and the trial function takes the above written simple form. The wave function is approximated as linear combination of the basis functions:

$$
\Phi(\mathbf{x})=\sum_{k=1}^{K} c_{k} f\left(A_{k}, \mathbf{x}\right)
$$

The ground state energy of this system is fairly accurately known by different calculations: $E=-0.262005$ a.u.. In Table 1.1 we illustrate what happens if completely random parameters are generated for various basis dimension $K=100,200$ and 400 . Five different random sets are generated (energies $E 1, E 2, E 3, E 4$ and $E 5$ to show how much the results scatter depending on the random parameters. The last line shows the number of function evaluations needed to reach these results. This measures the necessary computer time. Table 1.1 shows that even a completely random basis can lead to accurate results provided that the dimension is big enough. It also shows that the energy difference between different random sets is not too big and it actually decreases by increasing the basis size. Different random parameters give very similar results and not all of the random states are equally important. One may get almost the same result by omitting some of the states. One can, for example, sort out a smaller basis from the $K=400$ random basis. In the last column, under the title "best 100 " we have selected the 100 basis states which gives the lowest energy amongst the 400 randomly chosen one.

In the stochastic variational method random parameter sets are generated and we select the most appropriate set by comparing the energy gain. The basis is always built up step by step, increasing the dimension one-by-one, by adding a new set to the the previously fixed basis. The new set is chosen from several random candidates. Table 1.2 compares the energy obtained by the stochastic selection to that of the Powell optimization. The Powell optimization is a direct deterministic optimization of the parameters [16]. The fact that the Powell optimization gives slightly different results by starting from different random points shows that the omnipresence of the local minima makes such an optimization difficult. Table 1.2 also shows the result of SVM when we select amongst $n$ random candidate. The $n=1$ case means no selection but inclusion of random basis states, which is the same as in the case of Table 1.1 and it is included only for comparison. In the case of $n=10$ random 
Table 1.1. The energy of $\mathrm{Ps}^{-}$(in a.u.) for $K$ basis states that are selected randomly. "Exact" energy is -0.262005 . The energies $E i$ are obtained by starting from different random points. The energy of "Best 100" is the one calculated by sorting the best 100 basis states from 400 basis states, where 50 random trials are probed at each step of the basis selection. $N_{\text {eval }}$ is the number of matrix elements evaluated during the optimization.

\begin{tabular}{lllll}
\hline & $K=100$ & $K=200$ & $K=400$ & Best 100 \\
\hline$E 1$ & -0.2617619 & -0.2619798 & -0.2620032 & -0.2619995 \\
$E 2$ & -0.2617281 & -0.2619793 & -0.2620026 & -0.2619978 \\
$E 3$ & -0.2617918 & -0.2619669 & -0.2620016 & -0.2619956 \\
$E 4$ & -0.2618826 & -0.2619675 & -0.2620008 & -0.2619953 \\
$E 5$ & -0.2616285 & -0.2619824 & -0.2620024 & -0.2619987 \\
$N_{\text {eval }}$ & 5050 & 20100 & 80200 & 80200 \\
\hline
\end{tabular}

Table 1.2. Energy of $\mathrm{Ps}^{-}$(in a.u.) by different optimization strategies. The basis size is set to $K=100$. In the case of the full optimization by the Powell algorithm 7200 diagonalizations were required. The refining cycle is repeated only once.

\begin{tabular}{lllll}
\hline Method & Powell & $\operatorname{SVM}(n=1)$ & $\operatorname{SVM}(n=10)$ & Refining $(n=10)$ \\
\hline$E 1$ & -0.26200016 & -0.26176191 & -0.26199427 & -0.26200231 \\
$E 2$ & -0.26199947 & -0.26172812 & -0.26199382 & -0.26200351 \\
$E 3$ & -0.26200164 & -0.26179182 & -0.26199733 & -0.26200312 \\
$E 4$ & -0.26200135 & -0.26188261 & -0.26199778 & -0.26200301 \\
$E 5$ & -0.26200193 & -0.26162811 & -0.26199836 & -0.26200271 \\
$N_{\text {eval }}$ & 35466150 & 11615 & 86150 & 805050 \\
Time & 7200 & 27 & 43 & 195 \\
\hline
\end{tabular}

candidate, the results are very close to that of the Powell optimization, but the number of function evaluation, that is the computational load is more than two order of magnitudes smaller. After we reached a given dimension, we may stop increasing the basis size and we may try to replace the already selected basis states with a better choice. This is a refining of the basis and it is actually beats the results given by the Powell optimization, while the computational burden is still much smaller. The final result of the SVM is reached by repeating the refining steps and increasing the basis size until necessary/possible. Our most accurate calculation for this systems is compared to the results of other calculations in Table 1.3 
Table 1.3. Energy and different separation distances for the $\left(e^{+} e^{-} e^{-}\right)$Coulombic three-body system as a function of the basis dimension $K$. The virial ratio $\eta$ is defined by $\eta=|1+\langle V\rangle /(2\langle T\rangle)|$. Atomic units are used.

\begin{tabular}{lllll}
\hline & $\begin{array}{l}\text { SVM } \\
(K=100)\end{array}$ & $\begin{array}{l}\text { SVM } \\
(K=200)\end{array}$ & $\begin{array}{l}\text { SVM } \\
(K=600)\end{array}$ & $\begin{array}{l}\text { Hylleraas } \\
{[17]}\end{array}$ \\
\hline$-E$ & 0.26200465 & 0.2620050648 & 0.262005070226 & 0.2620050702328 \\
$\left\langle r_{+-}\right\rangle$ & 5.489 & 5.48962 & 5.489633252 & 5.489633252 \\
$\left\langle r_{--}\right\rangle$ & 8.548 & 8.54856 & 8.548580655 & 8.548580655 \\
$\left\langle r_{+-}^{2}\right\rangle^{\frac{1}{2}}$ & 6.958 & 6.95832 & 6.95837 & 6.95837 \\
$\left\langle r_{--}^{2}\right\rangle^{\frac{1}{2}}$ & 9.652 & 9.65284 & 9.65291 & 9.65291 \\
$\eta$ & $0.46 \times 10^{-4}$ & $0.34 \times 10^{-6}$ & $0.54 \times 10^{-10}$ & $0.23 \times 10^{-10}$ \\
\hline
\end{tabular}

\section{Excited states}

The Mini-Max theorem tells us that by diagonalizing the Hamiltonian in a $K$ dimensional basis one gets an upper bound not only for the ground state but also for the excited states as well. It may happen that the basis set found for the ground state is fairly good to predict the energies of the excited states with the same conserved quantum numbers (angular momentum, parity etc.) as the ground state. By optimizing only the ground state, however, the energies of the excited states will not necessarily converge.

As the $i$ th eigenvalue $E_{i}$ obtained by diagonalization is the upper bound of the energy of the $i$ th excited state, one may try to optimize this upper bound to get an accurate estimate for the energy of the excited state. In practice we repeat the same procedure as before, but now the basis selection is governed by the requirement that the $i$ th eigenvalue, not the ground-state energy $E_{1}$, should be improved. To get the $i$ th eigenvalue we need at least an $i$-dimensional basis to start with, but practical numerical considerations suggest that it is better to start with a basis in which all the lower eigenvalues $(k=1, \ldots, i-$ 1) are already "stable". This means that we need a first guess for the lower eigenvalues, otherwise it may happen that when improving the first excited state, for example, we pick up such components that lower the ground state.

As the energies of the excited states of the Helium atom are known to a high accuracy, we will test our strategy in this case. First we optimize the ground state of the He atom on a $K=100$-dimensional basis. The energy of the ground state is very accurate and even the energy of the first excited state is acceptable. Starting from this $K=100$-dimensional basis, we increase the basis size one by one, picking up basis states which improve the energy of the first excited state. This procedure quickly improves the energy of the first excited state, while the energy of the ground state also improves a little bit (the important thing to note is that it does not get worse) because the basis size is increased. After 
Table 2.1. Energies in atomic units of the ground state and the first four ${ }^{1} S$ excited states of the Helium atom. In column $A$ the basis is optimized successively for all the states as described in the text $(K=500)$. In column B the basis is optimized separately for each state, leading to five different bases $(K=600)$ tailored for the respective states. The "exact" values are taken from [18].

\begin{tabular}{llll}
\hline State & A & B & "Exact" \\
\hline$E_{1}$ & -2.9037243758 & -2.9037243769 & -2.90372437698 \\
$E_{2}$ & -2.1459737740 & -2.1459740452 & -2.14597404605 \\
$E_{3}$ & -2.0612718887 & -2.0612719880 & -2.06127198974 \\
$E_{4}$ & -2.0335865085 & -2.0335866779 & -2.03358671702 \\
$E_{5}$ & -2.0211312479 & -2.0211768312 & -2.02117685157 \\
\hline
\end{tabular}

reaching the basis size of $K=200$ we switch to the second excited state and so on.

The above procedure gives us a basis where all the excited states are accurate up to a certain digit. We can of course create bases which give an accurate energy for an individual state, while the energy of the other states might be poor. In that case we simply carry out the stochastic search for a given state starting from a first guess basis (like the $K=100$-dimensional basis in the previous case). This optimization may include refinement cycles as well, if necessary. The results we obtained in this way are compared to the "exact" (i.e., the best calculation in the literature) values [18] in Table 2.1 One can thus get as accurate energies for the excited states as for the ground state.

\section{Coulombic few-body systems}

The SVM has been widely applied for a number of Coulombic few-body problems. The real advantage of the method is that one can cope not only with 3 but 4-5-6 particles as well. It has been applied to few-electron atoms and for small molecules $[6,7]$. It made it possible to prove the existence of several positronic atoms, for the first time $[19,20]$. An extensive review can be found in Ref [2]. Some of our results are shown in Table 3.1 for illustration.

The $H_{3}^{+}$is a bound system but the system of three positrons and two electrons is found to be unbound [3]. It is intriguing to check at which mass ratio $m / M$ the $\left(M^{+}, M^{+}, M^{+}, m^{-}, m^{-}\right)$system looses the stability. The binding energy as a function of the mass ratio is shown in Fig. 1. The calculation shows that the stability is lost at around $m / M=0.22$. Around that point the fivebody system dissociates into a $\left(M^{+}, M^{+}, m^{-}, m^{-}\right)$four-body system (which is bound for any mass ratio $m / M$ ) and a $M^{+}$particle. It seems that up to that point the heavy particles can form a slowly moving stable frame which will break beyond that mass ratio. This can be very nicely seen in Fig. 2, where the average distances between the particles are shown as a function of the mass 
Table 3.1. Energies of different Coulombic systems in atomic units. $K$ is the basis dimension.

\begin{tabular}{lllllll}
\hline System & State & $K$ & SVM & Other method & $K$ & Ref. \\
\hline $\mathrm{Ps}_{2}$ & ${ }^{1} S^{e}$ & 800 & -0.516003778 & -0.516002 & 400 & {$[21]$} \\
$\mathrm{Ps}_{2}$ & ${ }^{1} P^{o}$ & 800 & -0.334408112 & & & \\
$\mathrm{Li}$ & ${ }^{1} S^{e}$ & 600 & -7.478058 & -7.47806032 & 1589 & {$[23]$} \\
$\mathrm{Li}$ & ${ }^{1} P^{o}$ & 1000 & -7.410151 & -7.410156521 & 1715 & {$[23]$} \\
$\mathrm{Li}$ & ${ }^{1} D^{e}$ & 1000 & -7.335520 & -7.335523540 & 1673 & {$[23]$} \\
${ }^{\infty} \mathrm{HPs}$ & ${ }^{1} S^{e}$ & 1200 & -0.7891964 & -0.7891794 & & {$[22]$} \\
$\mathrm{Be}$ & ${ }^{1} S^{e}$ & 500 & -14.6673 & -14.667355 & 1200 & {$[20]$} \\
$\mathrm{Li}^{-}$ & ${ }^{1} S^{e}$ & 600 & -7.50012 & -7.50076 & $\infty$ & {$[24]$} \\
$\mathrm{Li}+e^{+}$ & ${ }^{1} S^{e}$ & 1000 & -7.53218 & & & \\
$\mathrm{Li}+\mathrm{Ps}$ & ${ }^{1} S^{e}$ & 600 & -7.73855 & & & \\
$\mathrm{Be}+e^{+}$ & ${ }^{1} S^{e}$ & 1000 & -14.692 & & & \\
\hline
\end{tabular}

Table 4.1. Energies of few electron quantum dots calculated by SVM and by a large scale shell model (SM) basis. Atomic units are used. The harmonic oscillator frequency, $\omega=0.5$. The last column characterizes the shell model space used in the calculation.

\begin{tabular}{ccccc}
\hline$N(L, S, \pi)$ & $\mathrm{E}(\mathrm{SVM})$ & $\mathrm{K}$ & $\mathrm{E}(\mathrm{SM})$ & $\hbar \omega$ \\
\hline $3(1,1 / 2,-)$ & 4.01324 & 100 & 4.01324 & 39 \\
$4(0,0),+)$ & 6.35025 & 300 & 6.3506 & 14 \\
$5(1,1 / 2,-)$ & 9.00331 & 500 & 9.0032 & 6 \\
\hline
\end{tabular}

ratio.

\section{Confined few-electron systems}

In this section we consider a quadratically confined three-dimensional fewelectron system. This system can practically be viewed as an artificial atom, where the Coulombic attraction to the nucleus is replaced by a harmonic oscillator potential. Needless to say, the harmonic oscillator shell model type solutions are very succesful here because one only have to diagonalize the Coulombic repulsion between the electrons as the "residual interaction". We compare our results to a large scale shell model calculation [25] in Table 4.1 The energies are in very good agreement.

\section{Summary}

The stochastic variational method has proved to be useful in various fields of physics, including atomic, molecular, solid state, nuclear and subnuclear 


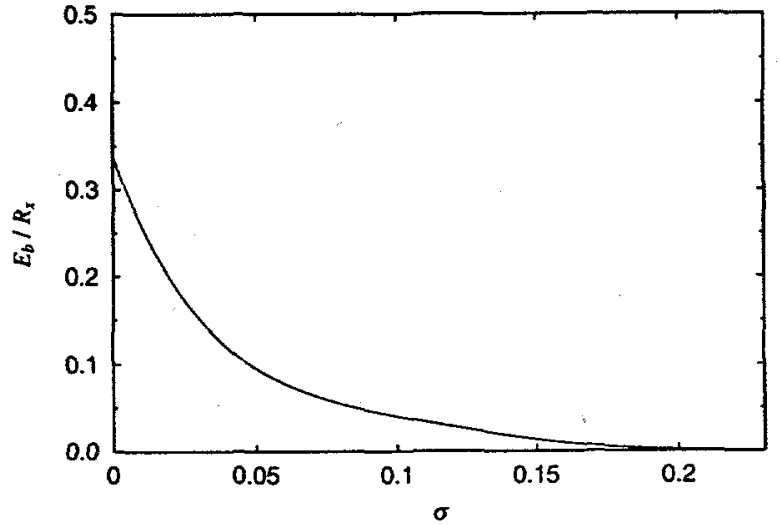

Figure 1. The binding energy of $\left(M^{+}, M^{+}, M^{+}, m^{-}, m^{-}\right)$as a function of mass ratio. The energy is measured in Rydberg.

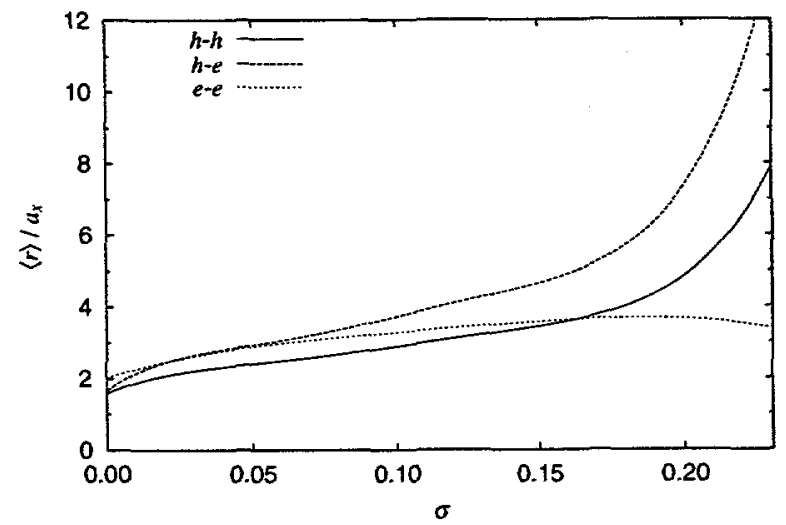

Figure 2. The average radius of $\left(M^{+}, M^{+}, M^{+}, m^{-}, m^{-}\right)$as a function of mass ratio the distance is measured in Bohr. 
physics. This paper only reviewed a small part of the applications. Other contributions to this volume will show its usefulness in studies related to the structure of the baryons $[26,27]$. Its main application is in nuclear physics, which has not been covered here but the interested reader can find examples in Refs. [10-13]. We would like to extend its applicability to larger systems and for more complicated interactions. Such developments are under way.

Acknowledgement. This work has been supported by OTKA grant No. T 17298 (Hungary) and Grants-in-Aid for Scientific Research (Nos. 08044065 and 10640255) of the Ministry of Education, Science and Culture (Japan). The work of $\mathrm{KV}$ is also supported by the U. S. Department of Energy, Nuclear Physics Division, under contract No. W-31-109-ENG-39. 


\section{References}

1. V. I. Kukulin and V. M. Krasnopol'sky, J. Phys. G: Nucl. Part. Phys. 3, 795 (1977).

2. Y. Suzuki and K. Varga, Stochastic variational approach to quantum mechanical few-body problems, Springer series on Lectures and Notes in Physics, in press.

3. K. Varga and Y. Suzuki, Phys. Rev. C52, 2885 (1995); Phys. Rev. A53, 1907 (1996).

4. Y. Suzuki, J. Usukura and K. Varga, J. Phys. B: At. Mol. Opt. Phys. 31, 31 (1998).

5. K. Varga and Y. Suzuki, Comp. Phys. Commun. 106, 157 (1997).

6. K. Varga, J. Usukura and Y. Suzuki, Phys. Rev. Lett. 80, 1876 (1998).

7. J. Usukura, K. Varga and Y. Suzuki, Phys. Rev. A, in press (1998).

8. Y. Suzuki, K. Varga and J. Usukura, Nucl. Phys. A631, 91c (1998).

9. K. Varga, Y. Suzuki and J. Usukura, Few-Body Systems 24, 81 (1998).

10. K. Varga, Y. Ohbayasi and Y. Suzuki, Phys. Lett. B396, 1 (1997).

11. K. Varga, Y. Suzuki and R. G. Lovas, Nucl. Phys. A371, 447 (1994).

12. K. Arai, Y. Suzuki and K. Varga, Phys. Rev. C51, 2488 (1995).

13. K. Varga, Y. Suzuki and I. Tanihata, Phys. Rev. C52, 3013 (1995).

14. S. F. Boys, Proc. R. Soc. London Ser. A258, 402 (1960).

15. K. Singer, Proc. R. Soc. London Ser. A258, 412 (1960).

16. W. H. Press, S. A. Teukolsky, W. T. Vetterling and B. P. Flanney, Numerical Recipes in FORTRAN, 2nd edition, (Cambridge University Press, New York, 1992).

17. Y. K. Ho, Phys. Rev. A48, 4780 (1993).

18. R. Krivec, M. I. Haftel and V. B. Mandelzweig, Phys. Rev. A44, 7158 (1991).

19. G. G. Ryzhikh and J. Mitroy, Phys. Rev. Lett. 79, 4124 (1997).

20. G. G. Ryzhikh, J. Mitroy and K. Varga, J. Phys. B: At. Mol. Opt. Phys. 31, L265 (1998).

21. A. M. Frolov, S. I. Kryuchkov and V. H. Smith, Jr., Phys. Rev. A 51, 4514 (1995). 
22. A. M. Frolov and V. H. Smith, Jr., J. Phys. B: At. Mol. Opt. Phys. 29, L433 (1996); Phys. Rev. A55, 2662 (1997).

23. Zhong-Chao Yan and G. W. F. Drake, Phys. Rev. A52, 3711 (1995).

24. C. Froese Fisher, J. Phys. B: At. Mol. Opt. Phys. 26, 855 (1993).

25. P. Navratil, Private communication.

26. L. Ya. Glozman, Z. Papp, W. Plessas, K. Varga and R. F. Wagenbrunn, Nucl. Phys. 623, 90 (1997); Phys. Rev. C57, 3406 (1998).

27. L. Ya. Glozman, W. Plessas, K. Varga and R. F. Wagenbrunn, Phys. Rev. $\mathrm{D}$, in press (1998). 